\title{
Associação entre o consumo energético e proteico e a espessura do músculo adutor do polegar em pacientes em hemodiálise
}

\author{
Association between energy and protein intake and the adductor pollicis muscle \\ thickness in hemodialysis patients
}

\author{
Alisson Diego Machado ${ }^{a}$, Camila Machado Rissotto ${ }^{b}$, Carmen Tzanno Branco Martinsc, \\ Ana Paula Bazanellid \\ a Nutricionista. Mestrando em Ciências pelo Programa de Pós-Graduação em Nefrologia da Universidade de São Paulo.

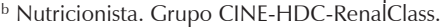 \\ c Médica. Doutora em Ciências pelo Programa de Pós-Graduação em Nefrologia da Universidade de São Paulo. Grupo CINE-HDC-RenalClass \\ d Nutricionista. Doutora em Ciências pelo Programa de Pós-Graduação em Nutrição da Universidade Federal de São Paulo. Universidade Presbiteriana Mackenzie.
}

RESUMO Introdução: A desnutrição energético-proteica é frequente em pacientes com doença renal crônica em hemodiálise e medidas alternativas para a avaliação do estado nutricional e do consumo alimentar podem ser empregadas na prática clínica.

Objetivo: Avaliar a associação entre o consumo energético e proteico e a espessura do músculo adutor do polegar em pacientes em hemodiálise.

Materiais e Métodos: Foi realizado um estudo transversal com 33 pacientes com doença renal crônica em hemodiálise. Foi aplicado um questionário para a identificação do perfil sociodemográfico dos pacientes. A ingestão de energia e de proteínas foi avaliada pela aplicação de dois recordatórios de 24 horas. A espessura do músculo adutor do polegar foi aferida com auxílio de um adipômetro. A associação entre as variáveis foi avaliada por meio de testes paramétricos, análise de correlação, regressão linear e curva ROC.

Resultados: A média de idade da amostra em estudo correspondeu a 68,0 anos, sendo 54,5\% do sexo masculino. Pacientes com idade inferior a 60 anos e do sexo masculino apresentaram uma maior espessura do músculo adutor do polegar $(p=0,01)$. Não houve associação entre a espessura do músculo adutor do polegar e o consumo energético e proteico. A medida também não é capaz de indicar quais pacientes apresentam uma maior ingestão de energia e proteínas.

Conclusão: Em pacientes com doença renal crônica em hemodiálise não houve associação entre o consumo energético e proteico e a espessura do músculo adutor do polegar e nem eficácia da medida em predizer uma maior ingestão de energia e proteínas.

Palavras-chave: consumo de alimentos; avaliação nutricional; diálise renal.

Introduction: Protein-energy wasting is common in patients with chronic kidney disease in hemodialysis and alternative measures for evaluation of the nutritional status and dietary intake may be employed in clinical practice.

Objective: To evaluate the association between energy and protein intake and the adductor pollicis muscle thickness in hemodialysis patients.

Materials and Methods: A cross-sectional study was conducted with 33 patients with chronic kidney disease in hemodialysis. A questionnaire to identify the sociodemographic profile of patients was applied. Energy and protein intake were evaluated by the application of two 24-hour recalls. Adductor pollicis muscle thickness was measured with a skinfold caliper. Association between variables was assessed using parametric tests, correlation analysis, linear regression and ROC curve.

Results: Mean age of the study sample corresponded to 68.0 years old and $54.5 \%$ were male. Patients aged less than 60 years old and males had a higher adductor pollicis muscle thickness $(p=0.01)$. There was no association between the adductor pollicis muscle thickness and energy and protein intake. The measure is also not able to indicate which patients have a higher energy and protein intake.

Conclusion: In patients with chronic kidney disease in hemodialysis there was no association between energy and protein intake and the adductor pollicis muscle thickness or effectiveness of the measure in predicting a higher energy and protein intake.

Keywords: food consumption; nutrition assessment; renal dialysis. 


\section{INTRODUÇÃO}

A doença renal crônica (DRC) é considerada um problema emergente de saúde pública mundial devido ao rápido aumento da sua prevalência nos últimos anos, com o agravante de que grande parte dos casos não é diagnosticada nas fases iniciais da doença ${ }^{1}$. Pode ser considerado portador de DRC o indivíduo que apresentar taxa de filtração glomerular menor que $60 \mathrm{~mL} / \mathrm{min} / 1,73 \mathrm{~m}^{2}$, ou com valor superior a essa referência, desde que associada a pelo menos um marcador de lesão renal parenquimatoso, presente há pelo menos três meses ${ }^{2}$, sendo o principal marcador utilizado a proteinúria ${ }^{3}$.

De acordo com censo realizado em 2013, as principais causas para o desenvolvimento da DRC na população brasileira foram a hipertensão arterial (35\%) e o diabetes mellitus (30\%). Estima-se que 100.397 pacientes estejam em terapia renal substitutiva no país, com uma taxa de prevalência de tratamento dialítico de 499 pacientes por milhão da população. O número de óbitos correspondeu a 17.944, correspondendo a uma taxa de mortalidade bruta de $17,9 \%$ durante o ano $^{4}$.

A perda de função renal provoca diversas alterações metabólicas, sendo a desnutrição energético-proteica (DEP) um dos distúrbios mais prevalentes em pacientes com DRC em hemodiálise, estando relacionada com o aumento das taxas de morbidade e mortalidade. Sua etiologia é multifatorial e inclui aspectos relacionados tanto ao consumo alimentar insuficiente em energia e nutrientes quanto às alterações hormonais e metabólicas que podem ocasionar um aumento do catabolismo energético e/ou proteico, como perda de nutrientes pelo dialisato, bioincompatibilidade das membranas dos filtros de diálise, resistência à ação da insulina e do hormônio do crescimento, acidose metabólica, presença de comorbidades e inflamação ${ }^{5}$, fazendo com que a avaliação nutricional seja de extrema importância no tratamento desses pacientes, sendo ideal a utilização de diferentes formas para essa avaliação ${ }^{6}$.

Nesse sentido, a utilização da espessura do músculo adutor do polegar (EMAP) é uma medida que passou recentemente a ser utilizada como marcador devido a ser facilmente aferida, além de não ser influenciada pelo estado hídrico ${ }^{7}$, sendo que alguns estudos demonstraram que a mesma é um bom marcador do estado nutricional em pacientes com DRC ${ }^{8,9}$. Embora alguns estudos tenham demonstrado bons resultados da utilização do EMAP na avaliação do estado nutricional, nenhum estudo demonstrou a sua relação com o consumo alimentar, especialmente o de energia e proteínas, já que uma das principais causas da DEP é a ingestão insuficiente de alimentos ${ }^{10,11}$.
Assim, o presente estudo teve por objetivo avaliar a associação entre o consumo energético e proteico e a EMAP em pacientes em hemodiálise.

\section{MATERIAIS E MÉTODOS}

Foi realizado um estudo transversal com 33 pacientes em hemodiálise - sessões de quatro horas, realizadas três vezes por semana - atendidos em uma clínica de nefrologia localizada no município de São Paulo-SP, durante agosto de 2015. A amostragem foi de conveniência, sendo inclusos pacientes com idade igual ou superior a 18 anos e que estavam em tratamento há pelo menos três meses. Foram excluídos pacientes que possuíam amputação de membros superiores ou inferiores ou qualquer enfermidade que impossibilitasse a aferição da EMAP.

Por meio de entrevista durante a sessão de diálise foi aplicado um questionário para a identificação do perfil sociodemográfico dos pacientes com perguntas referentes à idade, sexo, etnia, estado civil, turno e tempo de tratamento. Além disso, foram consultados no prontuário dos pacientes o peso seco atual e a estatura.

Após a entrevista foi aplicado um recordatório de 24 horas, que foi preenchido com todos os alimentos e bebidas consumidos no dia anterior por cada paciente, com auxílio do Álbum fotográfico de porções alimentares ${ }^{12}$. Além desse recordatório foi aplicado mais um em outro dia, para a avaliação do consumo alimentar durante o final de semana. Assim, foram avaliadas a ingestão de energia e de proteínas de dois dias, sendo um dia durante a semana e um de final de semana.

Após o preenchimento do primeiro recordatório de 24 horas foi aferida a EMAP. Para tanto, o paciente permaneceu sentado, sendo que o braço sem o acesso vascular foi mantido flexionado a aproximadamente $90^{\circ}$ com o antebraço, apoiado sobre a coxa e a mão sobre o joelho. Os pacientes foram orientados a permanecerem com a mão relaxada e o dedo polegar afastado do restante da mão, formando $90^{\circ}$ com o dedo indicador. Com auxílio de um adipômetro (Lange ${ }^{\circledR}$ ) foi exercida uma pressão de $10 \mathrm{~g} / \mathrm{mm}^{2}$ e o músculo foi pinçado no centro de um triângulo imaginário formado pelo dedo indicador e o polegar da mão. Essa medida foi aferida três vezes, por um único profissional devidamente treinado, sendo que foi utilizada a média das mesmas ${ }^{9}$.

Para a classificação do estado nutricional calculou-se o índice de massa corporal (IMC). Os pacientes com idade inferior a 60 anos foram classificados de acordo com o preconizado pela World Health Organization ${ }^{13}$, sendo IMC $<$ $16,0 \mathrm{~kg} / \mathrm{m}^{2}$ representa magreza grau III; $16,0-16,9 \mathrm{~kg} / \mathrm{m}^{2}$, magreza grau II; $17,0-18,4 \mathrm{~kg} / \mathrm{m}^{2}$, magreza grau I; $18,5-$ 
$24,9 \mathrm{~kg} / \mathrm{m}^{2}$, eutrofia; $25,0-29,9 \mathrm{~kg} / \mathrm{m}^{2}$, sobrepeso; 30,0 $34,5 \mathrm{~kg} / \mathrm{m}^{2}$, obesidade grau I; $35,0-39,9 \mathrm{~kg} / \mathrm{m}^{2} ; \mathrm{e} \geq 40,0 \mathrm{~kg} / \mathrm{m}^{2}$, obesidade grau III. Os pacientes com idade igual ou superior a 60 anos foram classificados segundo o proposto pela Organização Pan-Americana da Saúde ${ }^{14}$, sendo IMC $<23,0 \mathrm{~kg} / \mathrm{m}^{2}$ indica baixo peso; $23,0-27,9 \mathrm{~kg} / \mathrm{m}^{2}$, peso normal; $28,0-29,9 \mathrm{~kg} / \mathrm{m}^{2}$, sobrepeso; e $>30,0 \mathrm{~kg} / \mathrm{m}^{2}$, obesidade. Devido às diferenças nos pontos de corte estabelecidos, no presente estudo os pacientes que apresentaram magreza/baixo peso foram inclusos na categoria baixo peso; os que estavam na faixa de eutrofia/ peso normal na categoria peso adequado; e aqueles que apresentaram sobrepeso ou obesidade na categoria excesso de peso.

Foi calculado o peso ajustado dos pacientes que, de acordo com o IMC, apresentaram percentual de adequação do peso inferior a $95 \%$ ou superior a $115 \%$. Primeiramente foi calculado o peso ideal dos mesmos, de acordo com a seguinte fórmula: peso ideal $(\mathrm{kg})=\left[\mathrm{IMC}\right.$ desejadoxestatura $\left.(\mathrm{m})^{2}\right]$. O IMC desejado utilizado no estudo foi o IMC médio da classificação imediatamente anterior ao atual, no caso dos pacientes com sobrepeso ou obesidade; e o IMC médio imediatamente superior para os pacientes com baixo peso. A adequação do peso atual em relação ao ideal foi calculada a partir da seguinte equação: adequação do peso $(\%)=($ peso atual $\times 100$ )/peso ideal. O peso ajustado foi obtido por meio da fórmula proposta pela National Kidney Foundation (NKF) ${ }^{15}$ : peso ajustado $(\mathrm{kg})=[($ peso ideal - peso atual $) \times 0,25]+$ peso atual.

Os alimentos/preparações e bebidas consumidos foram convertidos em gramas, utilizando-se a Tabela para avaliação de consumo alimentar em medidas caseiras $^{16}$ e, quando o item não estava disponível na mesma, utilizou-se o Manual de avaliação do consumo alimentar em estudos populacionais: a experiência do inquérito de saúde em São Paulo (ISA) ${ }^{17}$. O consumo de energia e proteínas foi calculado pela utilização do software Nutrition Data System for Research ${ }^{\circledR}$. A ingestão de proteínas foi ajustada pela energia de acordo com o método dos resíduos, proposto por Willet e Stampfer ${ }^{18}$. O consumo foi comparado com a recomendação preconizada pela NKF ${ }^{15}$.

Foi aplicado o teste de Kolmogorov-Smirnov para verificação da normalidade dos dados. Uma vez que tanto a ingestão energética e proteica quanto a EMAP apresentaram distribuição normal, sua diferença de acordo com as variáveis analisadas foi avaliada pelos testes t de Student e análise de variância e os dados apresentados em média e desvio-padrão $(\mathrm{m} \pm \mathrm{dp})$. A correlação entre o consumo e a EMAP foi avaliada pelo coeficiente de correlação de Pearson. Foram estimados modelos de regressão linear para verificar a associação entre a ingestão de energia e proteínas e a EMAP. Além disso, para a avaliação da eficácia da EMAP em predizer um maior consumo energético e proteico, foi construída uma curva ROC (Receiver Operating Characteristic). Adotou-se nível de significância de 5\%. As análises estatísticas foram realizadas no software SPSS versão 17.0.

O estudo foi aprovado pelo Comitê de Ética em Pesquisa da Universidade Presbiteriana Mackenzie, sob número CAAE 51118515.7.0000.0084. Foram seguidas as determinações da Resolução no 466/2012, do Conselho Nacional de Saúde, sendo que a coleta de dados foi realizada após a assinatura do Termo de Consentimento Livre e Esclarecido por todos os participantes do estudo.

\section{RESULTADOS}

Foram inclusos 33 pacientes em hemodiálise, com média de idade de 68,0 $\pm 11,1$ anos (mínimo=43; máximo=88). As características sociodemográficas dos pacientes incluídos no estudo estão descritas na Tabela 1. Como pode ser observado, a maioria dos pacientes era idosa, do sexo masculino, de etnia branca e casada. A maior parte dos pacientes incluídos no estudo possuía peso adequado, realizava o tratamento no período vespertino e entre 3 e 15 meses.

Não houve diferença significativamente estatística no consumo de energia e de proteínas de acordo com as variáveis estudadas. Contudo, verificaram-se maiores valores da EMAP em pacientes com menos de 60 anos em relação aos pacientes com idade igual ou superior a 60, bem como para o sexo masculino em relação ao feminino (Tabela 1).

A ingestão energética média correspondeu a 15,0 $5,0 \mathrm{kcal} / \mathrm{kg} / \mathrm{dia}$ e a proteica a $0,8 \pm 0,2 \mathrm{~g} / \mathrm{kg} / \mathrm{dia}$, sendo que 32 pacientes $(97,0 \%)$ apresentaram um consumo inferior ao preconizado pela $\mathrm{NKF}^{15}$, tanto em energia quanto em proteínas, conforme demonstrado na Tabela 2.

Na Tabela 3 estão expressos a média e os percentis da EMAP dos pacientes incluídos no estudo, sendo a média

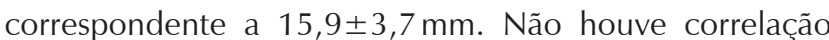
estatisticamente significativa entre a EMAP e a ingestão de energia $(r=-0,05 ; p=0,78)$, bem como entre o consumo de proteínas $(r=-0,01 ; p=0,95)$.

Considerando-se que houve diferença significativamente estatística entre pacientes adultos e idosos e do sexo masculino e feminino na medida da EMAP, foram estimados modelos de regressão linear ajustados pela idade, sexo ou ambos para verificar a sua associação entre a ingestão energética e proteica. Ainda que o valor de $p$ tenha reduzido após o ajuste, não houve associação significativa entre as variáveis (Tabela 4). 
Tabela 1. Distribuição do consumo energético e proteico e da EMAP de pacientes em hemodiálise de acordo com variáveis sociodemográficas e de tratamento.

\begin{tabular}{|c|c|c|c|c|c|}
\hline Variável & $\mathbf{n}$ & $\%$ & $\begin{array}{c}\text { Consumo energético } \\
\text { (kcal/kg/dia) } \\
\mathbf{m} \pm \mathbf{d p}\end{array}$ & $\begin{array}{c}\text { Consumo proteico } \\
\text { (g/kg/dia) } \\
\mathbf{m} \pm \mathrm{dp}\end{array}$ & $\begin{array}{l}\text { EMAP } \\
(\mathrm{mm}) \\
\mathrm{m} \pm \mathrm{dp}\end{array}$ \\
\hline \multicolumn{6}{|l|}{ Idade } \\
\hline$<60$ anos & 6 & 18,2 & $17,3 \pm 2,7$ & $0,8 \pm 0,2$ & $19,4 \pm 4,1^{*}$ \\
\hline$\geq 60$ anos & 27 & 81,8 & $14,4 \pm 5,3$ & $0,8 \pm 0,2$ & $15,1 \pm 3,2$ \\
\hline \multicolumn{6}{|l|}{ Sexo } \\
\hline Masculino & 18 & 54,5 & $15,5 \pm 5,3$ & $0,8 \pm 0,2$ & $17,4 \pm 3,4^{*}$ \\
\hline Feminino & 15 & 45,5 & $14,3 \pm 4,8$ & $0,8 \pm 0,2$ & $14,1 \pm 3,3$ \\
\hline \multicolumn{6}{|l|}{ Etnia } \\
\hline Branca & 18 & 54,5 & $14,3 \pm 5,6$ & $0,8 \pm 0,2$ & $15,1 \pm 3,3$ \\
\hline Não-branca & 15 & 45,5 & $15,8 \pm 4,4$ & $0,8 \pm 0,2$ & $16,9 \pm 3,9$ \\
\hline \multicolumn{6}{|l|}{ Estado civil } \\
\hline Com cônjuge & 17 & 51,5 & $16,6 \pm 5,0$ & $0,8 \pm 0,2$ & $16,3 \pm 3,7$ \\
\hline Sem cônjuge & 16 & 48,5 & $13,2 \pm 4,6$ & $0,9 \pm 0,2$ & $15,6 \pm 3,8$ \\
\hline \multicolumn{6}{|l|}{ Estado nutricional } \\
\hline Baixo peso & 5 & 15,2 & $17,4 \pm 10,2$ & $0,9 \pm 0,1$ & $12,4 \pm 2,1$ \\
\hline Peso adequado & 15 & 45,5 & $14,5 \pm 3,5$ & $0,8 \pm 0,2$ & $16,7 \pm 2,4$ \\
\hline Excesso de peso & 13 & 39,4 & $14,5 \pm 4,0$ & $0,8 \pm 0,2$ & $16,4 \pm 4,7$ \\
\hline \multicolumn{6}{|l|}{ Turno de tratamento } \\
\hline Matutino & 10 & 30,3 & $14,5 \pm 3,6$ & $0,7 \pm 0,1$ & $16,1 \pm 3,6$ \\
\hline Vespertino & 14 & 42,4 & $16,3 \pm 6,5$ & $0,9 \pm 0,2$ & $15,9 \pm 3,3$ \\
\hline Noturno & 9 & 27,3 & $13,5 \pm 3,7$ & $0,8 \pm 0,2$ & $15,8 \pm 4,7$ \\
\hline \multicolumn{6}{|l|}{ Tempo de tratamento } \\
\hline 3 a 15 meses & 13 & 39,4 & $16,7 \pm 6,0$ & $0,8 \pm 0,2$ & $15,2 \pm 2,9$ \\
\hline 15 a 27 meses & 8 & 24,2 & $14,3 \pm 3,8$ & $0,9 \pm 0,1$ & $17,1 \pm 3,1$ \\
\hline 27 meses ou mais & 12 & 36,4 & $13,5 \pm 4,4$ & $0,8 \pm 0,1$ & $16,0 \pm 4,7$ \\
\hline
\end{tabular}

EMAP: espessura do músculo adutor do polegar; Sem cônjuge: solteiro(a), viúvo(a) ou divorciado(a)/separado(a) judicialmente. m: média; dp: desvio padrão. ${ }^{*} p=0,01$ na medida da EMAP.

Tabela 2. Média, percentis de consumo e prevalência de inadequação do consumo energético e proteico de pacientes em hemodiálise.

\begin{tabular}{|c|c|c|c|c|c|c|c|}
\hline \multirow{2}{*}{ Variável } & \multirow{2}{*}{ Média } & \multicolumn{5}{|c|}{ Percentis de consumo } & \multirow{2}{*}{$\begin{array}{c}\text { Prevalência de } \\
\text { inadequação (\%) }\end{array}$} \\
\hline & & 10 & 25 & 50 & 75 & 90 & \\
\hline Energia (kcal/kg/dia) & 15,0 & 9,5 & 10,9 & 14,7 & 17,5 & 20,4 & 97,0 \\
\hline Proteína (g/kg/dia) & 0,8 & 0,6 & 0,7 & 0,8 & 0,9 & 1,1 & 97,0 \\
\hline
\end{tabular}

Tabela 3. Média e percentis da EMAP de pacientes em hemodiálise.

\begin{tabular}{|c|c|c|c|c|c|c|}
\hline \multirow{2}{*}{ Variável } & \multirow{2}{*}{ Média } & \multicolumn{5}{|c|}{ Percentis } \\
\hline & & 10 & 25 & 50 & 75 & 90 \\
\hline EMAP (mm) & 15,9 & 11,2 & 13,0 & 15,5 & 19,0 & 20,0 \\
\hline
\end{tabular}

EMAP: espessura do músculo adutor do polegar.

Tabela 4. Associação entre o consumo energético e proteico e a EMAP de pacientes em hemodiálise.

\begin{tabular}{|c|c|c|c|}
\hline Variável & $\beta$ & IC95\% & $p$ \\
\hline \multicolumn{4}{|l|}{ Modelo 1 - Modelo bruto } \\
\hline Consumo de energia (kcal/kg/dia) & $-0,04$ & $-0,31-0,23$ & 0,78 \\
\hline Consumo de proteínas (g/kg/dia) & $-0,23$ & $-7,70-7,24$ & 0,95 \\
\hline \multicolumn{4}{|l|}{ Modelo 2 - Ajustado para idade e sexo } \\
\hline Consumo de energia $(\mathrm{kcal} / \mathrm{kg} / \mathrm{dia})$ & $-0,07$ & $-0,27-0,12$ & 0,45 \\
\hline Consumo de proteínas (g/kg/dia) & 3,73 & $-1,66-9,12$ & 0,17 \\
\hline \multicolumn{4}{|c|}{ Modelo 3 - Ajustado para idade, sexo e consumo energético } \\
\hline Consumo de proteínas (g/kg/dia) & 4,18 & $-1,29-9,64$ & 0,13 \\
\hline
\end{tabular}

EMAP: espessura do músculo adutor do polegar. 

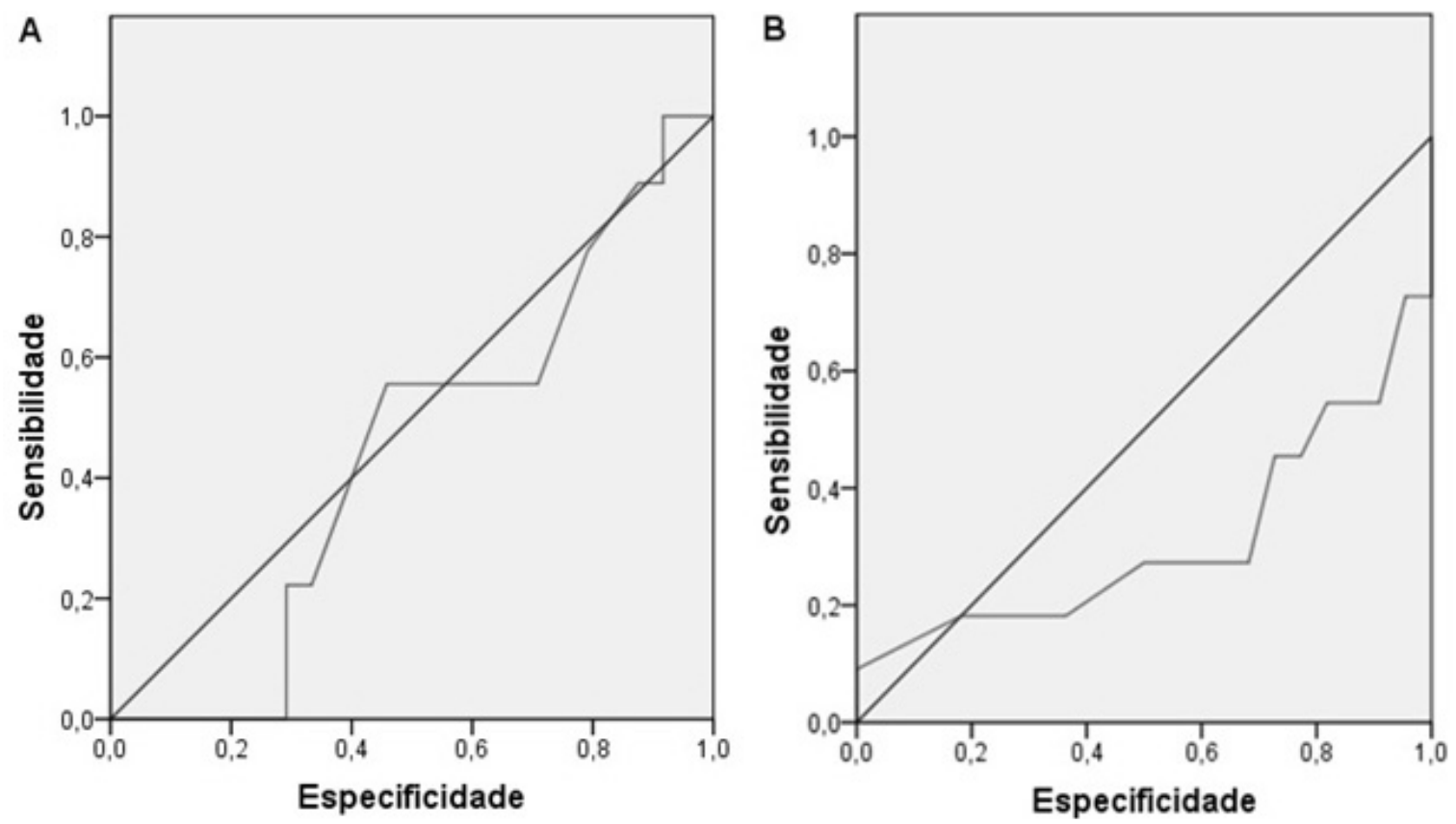

Figura 1. Área sob a curva, sensibilidade e especificidade da espessura do músculo adutor do polegar (EMAP) em predizer um maior consumo energético (A) e proteico (B).

Para a predição da eficácia da EMAP em identificar os pacientes que consumiram uma maior quantidade de energia e de proteínas ( $\geq p 75)$, foi construída uma curva ROC para cada variável. Verificou-se uma área sob a curva de 0,44 (IC95\% 0,23-0,65; $p=0,61$ ) para o consumo de energia e de 0,31 (IC95\% 0,09-0,53; $p=0,08$ ) para o de proteínas, demonstrando que a medida não é capaz de predizer quais pacientes estão classificados no quartil mais alto de ingestão energética e proteica (Figura 1).

\section{DISCUSSÃO}

A maioria dos pacientes incluídos no estudo apresentou um consumo, tanto de energia quanto de proteínas, inferior ao preconizado pela NKF ${ }^{15}$, que recomenda a ingestão de energia de $35 \mathrm{kcal} / \mathrm{kg} /$ dia para pacientes com até 59 anos e de $30-35 \mathrm{kcal} / \mathrm{kg} /$ dia para aqueles com idade igual ou superior a 60 anos, e um consumo proteico de 1,2 g/ $\mathrm{kg} / \mathrm{dia}$. Pinto et al. ${ }^{19}$ identificaram uma ingestão energética e proteica correspondente a $28,0 \mathrm{kcal} / \mathrm{kg} / \mathrm{dia}$ e $1,1 \mathrm{~g} / \mathrm{kg} / \mathrm{dia}$, respectivamente, em pacientes em hemodiálise, ao passo que Machado et al. ${ }^{20}$ verificaram um consumo de energia equivalente a $19,0 \mathrm{kcal} / \mathrm{kg} /$ dia e de proteínas a $0,9 \mathrm{~g} / \mathrm{kg} / \mathrm{dia}$, também em pacientes com DRC em hemodiálise. Embora tais valores sejam maiores em relação ao presente estudo, também estão abaixo do recomendado para essa população, demonstrando a baixa ingestão energética e proteica nesses pacientes.
Antunes et al. ${ }^{21}$, ao avaliarem a influência dos parâmetros nutricionais sobre a sobrevida de pacientes em diálise, demonstraram que o consumo inferior a $1,2 \mathrm{~g} / \mathrm{kg} / \mathrm{dia}$ de proteínas constitui-se como um preditor independente de mortalidade nos mesmos. Por sua vez, Naylor et al. ${ }^{22}$, ao realizarem uma revisão de literatura com o objetivo de elaborar diretrizes sobre a necessidade de proteínas de pacientes em hemodiálise, chegaram à conclusão de que a ingestão de $1,1 \mathrm{~g} / \mathrm{kg} /$ dia desse macronutriente é capaz de atender as necessidades dos mesmos e evitar a desnutrição proteica. Contudo, ainda assim o consumo de proteínas demonstrou-se abaixo desse valor na amostra em estudo.

Em relação à EMAP, em estudo realizado por Oliveira et al. ${ }^{8}$, identificou-se uma espessura média de $12,3 \mathrm{~mm}$ para o sexo masculino e $11,2 \mathrm{~mm}$ para o sexo feminino, com diferença significativamente estatística entre ambos. Em consoante com esse achado, o presente estudo também verificou maiores valores para o sexo masculino em relação ao feminino, embora no presente estudo os pacientes tenham apresentado maiores medidas em relação ao de Oliveira et al. ${ }^{8}$. Além disso, verificou-se também que os pacientes com menos de 60 anos apresentaram uma maior espessura em relação aos pacientes com idade igual ou superior a 60 anos. Por sua vez, Pereira et al. ${ }^{9}$ identificaram uma mediana de $10 \mathrm{~mm}$ da EMAP em pacientes em hemodiálise, sendo que os pacientes mais jovens também apresentaram maiores valores nesse parâmetro. 
O presente estudo caracteriza-se como o primeiro que avaliou a associação entre a EMAP e o consumo alimentar. Pereira et al. $^{9}$ verificaram que não houve relação entre a EMAP e o equivalente proteico do aparecimento de nitrogênio (PNA), que se caracteriza como uma medida bioquímica utilizada para estimar a ingestão de proteínas ${ }^{23}$, o que sugere que realmente não há associação entre a EMAP e o consumo proteico, uma vez que no presente estudo verificou-se que não havia associação mesmo quando houve o ajuste por idade, sexo ou ambos, bem como pelo consumo energético.

Embora nenhum estudo tenha avaliado a associação entre a ingestão de energia e de proteínas, diferentes estudos realizados com a EMAP e sua associação com a antropometria de pacientes em hemodiálise verificaram diferentes resultados ${ }^{8,9}$, embora essa medida pareça estar relacionada com o estado nutricional em menor ou maior grau. As limitações relacionadas à EMAP incluem a falta de valores de referência e o fato de não haver estudos que avaliaram a reprodutibilidade da medida intra e interobservador ${ }^{9}$.

Como fatores limitantes do presente estudo destacam-se o número de participantes relativamente baixo e o consumo homogêneo entre os participantes. Além disso, a própria amostra era homogênea, composta majoritariamente por idosos, o que pode enfraquecer o achado de diferença entre essas fases da vida em relação à medida da EMAP. Também deve ser considerado que a oferta e a qualidade da hemodiálise e o acompanhamento nutricional realizado na clínica podem ter influenciado nos resultados do estudo.

Assim, verificou-se que em pacientes com DRC em hemodiálise não houve associação entre o consumo energético e proteico e a EMAP, nem eficácia deste último em predizer uma maior ingestão de energia e proteínas.

\section{REFERÊNCIAS}

1. Kirsztajn GM, Bastos MG, Andriolo A. Dia Mundial do Rim 2011Proteinúria e creatinina sérica: testes essenciais para diagnóstico de doença renal crônica. J Bras Patol Med Lab. 2011;47:100-3. https:// doi.org/10.1590/S1676-24442011000200002

2. National Kidney Foundation. Kidney Disease Outcomes Quality Initiative. Clinical practice guidelines for chronic kidney disease: evaluation, classification and stratification. Am J Kidney Dis. 2002; 39:S1-266

3. Bastos MG, Kirsztajn GM. Doença renal crônica: importância do diagnóstico precoce, encaminhamento imediato e abordagem multidisciplinar estruturada para melhora do desfecho em pacientes ainda não submetidos à diálise. J Bras Nefrol. 2011;33:93-108. https://doi.org/10.1590/S0101-28002011000100013

4. Sesso RC, Lopes AA, Thomé FS, Lugon JR, Santos DR. Inquérito Brasileiro de Diálise Crônica 2013 - Análise das tendências entre 2011 e 2013. J Bras Nefrol. 2014;36:476-81. https://doi. org/10.5935/0101-2800.20140068
5. Nerbass FB, Cuppari L. Hemodiálise. In: Cuppari L, Avesani CM, Kamimura MA. Nutrição na doença renal crônica. Barueri: Manole; 2013. p. 247-69.

6. Segall L, Moscalu M, Hogas S, Mititiuc I, Nistor I, Veisa G, Covic A. Protein-energy wasting, as well as overweight and obesity, is a long-term risk factor for mortality in chronic hemodialysis patients. Int Urol Nephrol. 2014;46:615-21. https://doi.org/10.1007/s11255014-0650-0

7. Gonzalez MC, Duarte RRP, Budziareck MB. Adductor pollicis muscle: reference values of its thickness in a healthy population. Clin Nutr. 2010;29:268-71. https://doi.org/10.1016/j.clnu.2009.08.012

8. Oliveira CMC, Kubrusly M, Mota RS, Choukroun G, Brandão Neto J, Silva CAB. Adductor pollicis muscle thickness: a promising anthropometric parameter for patients with chronic renal failure. J Ren Nutr. 2012;22:307-16. https://doi.org/10.1053/j. jrn.2011.07.006

9. Pereira RA, Caetano AL, Cuppari L, Kamimura MA. Espessura do músculo adutor do polegar como preditor da força de preensão manual nos pacientes em hemodiálise. J Bras Nefrol. 2013;35: 177-84. https://doi.org/10.5935/0101-2800.20130029

10. Riella MC. Nutritional evaluation of patients receiving dialysis for the management of protein-energy wasting: what is old and what is new? J Ren Nutr. 2013;23:195-8. https://doi.org/10.1053/j. jirn.2013.01.023

11. Ravel VA, Molnar MZ, Streja E, Kim JC, Victoroff A, Jing J, Benner D, Norris KC, Kovesdy CP, Kopple JD, Kalantar-Zadeh K. Low protein nitrogen appearance as a surrogate of low dietary protein intake is associated with higher all-cause mortality in maintenance hemodialysis patients. J Nutr. 2013;143:1084-92. https://doi. org/10.3945/jn.112.169722

12. Lopez RPS, Botelho RBA. Álbum fotográfico de porções alimentares. São Paulo: Metha; 2013.

13. World Health Organization. Obesity: preventing and managing the global epidemic. Geneva: WHO; 2000.

14. Organização Pan-Americana da Saúde. XXXVI Reunión del Comitê Asesor de Investigaciones en Salud - Encuestra Multicêntrica - Salud Beinestar y Envejecimiento (SABE) en América Latina e el Caribe - Informe Preliminar [cited 2015 Ago 25]. 2002. Available from: http://www.opas.org/program/sabe.htm

15. National Kidney Foundation. Kidney Disease Outcomes Quality Initiative. Clinical practice guidelines for nutrition in chronic renal failure. Am J Kidney Dis. 2000;35:S1-140.

16. Pinheiro ABV, Lacerda EMA, Benzecry EH, Gomes MCS, Costa VM. Tabela para avaliação de consumo alimentar em medidas caseiras. 5ae ed. São Paulo: Atheneu; 2008.

17. Fisberg RM, Marchioni DML. Manual de avaliação do consumo alimentar em estudos populacionais: a experiência do inquérito de saúde em São Paulo (ISA). São Paulo: Faculdade de Saúde Pública da USP; 2012.

18. Willett WC, Stampfer MJ. Total energy intake: implications for epidemiological analyses. Am J Epidemiol. 1986;124:17-27.

19. Pinto DE, Ullmann LS, Burmeister MM, Antonello ICF, Pizzato A. Associações entre ingestão energética, proteica e de fósforo em pacientes portadores de doença renal crônica em tratamento hemodialítico. J Bras Nefrol. 2009;31:269-74. https://doi.org/ 10.1590/S0101-28002009000400005 
20. Machado AD, Bazanelli AP, Simony RF. Avaliação do consumo alimentar de pacientes com doença renal crônica em hemodiálise. Ciênc Saúde. 2014;7:76-84. https://doi.org/10.15448/1983$652 X .2014 .2 .17758$

21. Antunes AA, Vannini FD, Silveira LVA, Martin LC, Barretti P, Caramori $J C$. Influence of protein intake and muscle mass on survival in chronic dialysis patients. Ren Fail. 2010;32:1055-9. https://doi.or g/10.3109/0886022X.2010.510233

22. Naylor HL, Jackson H, Walker GH, Macafee S, Magee K, Hooper L, Stewart L, MacLaughlin HL; Renal Nutrition Group of the British
Dietetic Association, British Dietetic Association. British Dietetic Association evidence-based guidelines for the protein requirements of adults undergoing maintenance haemodialysis or peritoneal dialysis. J Hum Nutr Diet. 2013;26:315-28. https://doi.org/10.1111/ jhn.12052

23. Velludo CM, Kamimura MA, Moreira PFP, Avesani CM, Ribeiro FSM, Vasselai P, Manfredi SR, Canziani MEF, Draibe AS, Cuppari L. Estimativa de ingestão proteica de pacientes em hemodiálise: comparação entre registro alimentar e equivalente proteico de aparecimento de nitrogênio (PNA). J Bras Nefrol. 2007;29:245-51. 